\title{
COMPARISON THEOREMS FOR NONSELFADJOINT DIFFERENTIAL EQUATIONS BASED ON INTEGRAL INEQUALITIES
}

\author{
KURT KREITH
}

Abstract. In a variant of the classical Sturmian comparison theorem for selfadjoint Sturm-Liouville equations, A. Ju. Levin has replaced the pointwise conditions on the coefficients by an integral inequality. This theorem is generalized to apply to nonselfadjoint differential equations of the form

$$
u^{\prime \prime}+b(x) u^{\prime}+c(x) u=0 .
$$

The basic Sturmian comparison theorem deals with functions $u(x)$ and $v(x)$ satisfying

$$
\begin{gathered}
u^{\prime \prime}+c(x) u=0, \\
v^{\prime \prime}+\gamma(x) v=0 .
\end{gathered}
$$

If $\gamma(x) \geqq c(x)$, then solutions of (2) oscillate more rapidly than solutions of (1). More precisely, if $u(x)$ is a nontrivial solution of (1) for which $u\left(x_{1}\right)=u\left(x_{2}\right)=0\left(x_{1}<x_{2}\right)$ and $\gamma(x) \geqq c(x)$ for $x_{1} \leqq x \leqq x_{2}$, then $v(x)$ has a zero in $\left(x_{1}, x_{2}\right]$.

This basic result has numerous generalizations, the following of which will be relevant to this paper.

1. It is possible to replace the condition $u\left(x_{1}\right)=0$ by $u^{\prime}\left(x_{1}\right)+\sigma u\left(x_{1}\right)=0$ where $\sigma$ is a constant $(-\infty \leqq \sigma<\infty)$ and $\sigma=-\infty$ is used to denote the condition $u\left(x_{1}\right)=0$. In this case one concludes that every solution of (2) satisfying $v^{\prime}\left(x_{1}\right)+\tau v\left(x_{1}\right)=0$ with $\tau \geqq \sigma$ has a zero in $\left(x_{1}, x_{2}\right]$.

2 . The pointwise inequality $\gamma(x) \geqq c(x)$ can be replaced by weaker integral inequalities. Such weaker conditions have been established by several authors, but of special interest to us here are the results of Levin [1] which deal with nontrivial solutions of (1) and (2) satisfying

$$
\begin{gathered}
u^{\prime}\left(x_{1}\right)+\sigma u\left(x_{1}\right)=0, \\
v^{\prime}(x)+\tau v\left(x_{1}\right)=0,
\end{gathered}
$$

respectively.

Received by the editors July 26, 1971.

AMS 1969 subject classifications. Primary 3442.

Key nords and phrases. Comparison theorem, Riccati equations, integral equation.

(c) American Mathematical Society 1972 
TheOREM 1 (LeVin). If $\sigma$ and $\tau$ are finite, $u\left(x_{2}\right)=0$ for some $x_{2}>x_{1}$, and if

$$
\tau+\int_{x_{1}}^{x} \gamma(t) d t \geqq\left|\sigma+\int_{x_{1}}^{x} c(t) d t\right|
$$

for $x_{1} \leqq x \leqq x_{2}$, then $v(x)$ has a zero in $\left(x_{1}, x_{2}\right]$.

3. Sturmian theorems have been studied for the general nonselfadjoint linear differential equation of second order. Such results have been established by Kamke [2] using the Pruefer transformation and by Swanson [3] and the author [4] as special cases of Sturmian theorems for nonselfadjoint elliptic equations. However the hypotheses required in these theorems were either pointwise inequalities or integral inequalities of a type different from Levin's and involving the solution $u(x)$ of the differential equation which "oscillates slower.'

The purpose of this paper is to unify the three generalizations indicated above for the case of nonselfadjoint equations of the form

$$
\begin{aligned}
& u^{\prime \prime}-2 b(x) u^{\prime}+c(x) u=0, \\
& v^{\prime \prime}-2 \beta(x) v^{\prime}+\gamma(x) v=0
\end{aligned}
$$

whose coefficients are assumed to be real and continuous. (The general linear second order differential equation can always be put into such form by use of a Liouville transformation.) By means of the transformation

$$
w=-u^{\prime} / u ; \quad z=-v^{\prime} / v ;
$$

the equations (5) and (6) are transformed into Riccati equations

$$
\begin{aligned}
w^{\prime} & =w^{2}+2 b w+c, \\
z^{\prime} & =z^{2}+2 \beta z+\gamma
\end{aligned}
$$

and the initial conditions

$$
-u^{\prime}\left(x_{1}\right) / u\left(x_{1}\right)=\sigma ; \quad-v^{\prime}\left(x_{1}\right) / v\left(x_{1}\right)=\tau
$$

for (5) and (6) become initial values

$$
w\left(x_{1}\right)=\sigma ; \quad z\left(x_{1}\right)=\tau
$$

for $\left(5^{\prime}\right)$ and $\left(6^{\prime}\right)$. The differential equations $\left(5^{\prime}\right)$ and $\left(6^{\prime}\right)$ subject to $(8)$ can in turn be written as integral equations

$$
\begin{aligned}
& w(x)=\sigma+\int_{x_{1}}^{x} w^{2} d t+\int_{x_{1}}^{x} 2 b w d t+\int_{x_{1}}^{x} c d t, \\
& z(x)=\tau+\int_{x_{1}}^{x} z^{2} d t+\int_{x}^{x} 2 \beta z d t+\int_{x_{1}}^{x} \gamma d t .
\end{aligned}
$$


It is obvious from these equations that if $\tau \geqq \sigma \geqq 0, \beta(x) \geqq b(x) \geqq 0$, and

$$
\int_{x_{1}}^{x} \gamma(t) d t \geqq \int_{x_{1}}^{x} c(t) d t \geqq 0
$$

on an interval $\left[x_{1}, x_{2}\right]$, then $z(x) \geqq w(x) \geqq 0$ as long as $z(x)$ can be continued on $\left[x_{1}, x_{2}\right]$. Since the singularities of $w(x)$ and $z(x)$ correspond to the zeros of $u(x)$ and $v(x)$, respectively, these observations lead to the following elementary comparison theorem for (5) and (6).

THEOREM 2. Suppose $u(x)$ is a nontrivial solution of (5) satisfying $-u^{\prime}\left(x_{1}\right) / u\left(x_{1}\right)=\sigma \geqq 0, u\left(x_{2}\right)=0$. If

$$
\begin{aligned}
& \beta(x) \geqq b(x) \geqq 0 \quad \text { for } x_{1} \leqq x \leqq x_{2}, \\
& \int_{x_{1}}^{x} \gamma(t) d t \geqq \int_{x_{1}}^{x} c(t) d t \geqq 0 \text { for } x_{1} \leqq x \leqq x_{2} \text {, }
\end{aligned}
$$

then every solution of (6) satisfying $-v^{\prime}\left(x_{1}\right) / v\left(x_{1}\right) \geqq \sigma$ has a zero in $\left(x_{1}, x_{2}\right]$.

We shall be interested in variations of Theorem 2 which do not require the nonnegativity of $\sigma, \tau, b(x)$, and $\int_{x_{1}}^{x} c(t) d t$. To that end we note that the integral equations $\left(5^{\prime \prime}\right)$ and $\left(6^{\prime \prime}\right)$ can be written

$$
\begin{aligned}
& w(x)=\sigma+\int_{x_{1}}^{x}(w+b)^{2} d t+\int_{x_{1}}^{x}\left(c-b^{2}\right) d t, \\
& z(x)=\tau+\int_{x_{1}}^{x}(z+\beta)^{2} d t+\int_{x_{1}}^{x}\left(\gamma-\beta^{2}\right) d t .
\end{aligned}
$$

This formulation shows that condition (ii) of Theorem 2 can be replaced by

$$
\int_{x_{1}}^{x}\left(\gamma-\beta^{2}\right) d t \geqq \int_{x_{1}}^{x_{2}}\left(c-b^{2}\right) d t \geqq 0 .
$$

It also allows for other results of a more general nature.

LEMMA 1. Let $w(x)$ and $z(x)$ be solutions of $\left(5^{\prime \prime}\right)$ and $\left(6^{\prime \prime}\right)$, respectively, for which $\sigma>-\infty$ and

(i) $\tau+\int_{x_{1}}^{x}\left(\gamma-\beta^{2}\right) d t>\left|\sigma+\int_{x_{1}}^{x}\left(c-b^{2}\right) d t\right|$ for $x_{1} \leqq x \leqq x_{2}$,

$$
\beta(x) \geqq|b(x)| \quad \text { for } x_{1} \leqq x \leqq x_{2} .
$$

Then $z(x)>|w(x)|$ as long as $z(x)$ can be continued on $\left[x_{1}, x_{2}\right]$.

Proof. From $\left(6^{\prime \prime}\right)$ we have $z(x) \geqq \tau+\int_{x_{1}}^{x}\left(\gamma-\beta^{2}\right) d t$ for $x_{1} \leqq x \leqq x_{2}$. Using 
(i) and $\left(5^{\prime \prime}\right)$ this implies that

$$
\begin{aligned}
z(x) & >-\sigma-\int_{x_{1}}^{x}\left(c-b^{2}\right) d t \\
& >-\sigma-\int_{x_{1}}^{x}\left(c-b^{2}\right) d t-\int_{x_{1}}^{x}(w+b)^{2} d t>-w(x)
\end{aligned}
$$

for $x_{1} \leqq x \leqq x_{2}$. It remains to show that $z(x)>w(x)$. To that end we assume to the contrary that there exists $x_{0} \in\left(x_{1}, x_{2}\right]$ such that $z\left(x_{0}\right) \leqq w\left(x_{0}\right)$. Then there exists an $\tilde{x} \in\left(x_{1}, x_{0}\right]$ such that $z(\tilde{x})=w(\tilde{x})$ and $z(x)>|w(x)|$ for $x_{1} \leqq x<\tilde{x}$. Using (ii) we have that

$$
z(x)+\beta(x)>|w(x)|+|b(x)| \geqq|w(x)+b(x)| \text { for } x_{1} \leqq x<\tilde{x},
$$

and consequently that $\int_{x_{1}}^{\tilde{x}}(z+\beta)^{2} d t>\int_{x_{1}}^{\tilde{x}}(w+b)^{2} d t$. Using $\left(6^{\prime \prime}\right)$, (i), and $\left(5^{\prime \prime}\right)$ yields

$$
\begin{aligned}
w(\tilde{x}) & =\sigma+\int_{x_{1}}^{\tilde{x}}\left(c+b^{2}\right) d t+\int_{x_{1}}^{\tilde{x}}(w+b)^{2} d t \\
& <\tau+\int_{x_{1}}^{\tilde{x}}\left(\gamma-\beta^{2}\right) d t+\int_{x_{1}}^{\tilde{x}}(z+\beta)^{2} d t=z(\tilde{x})
\end{aligned}
$$

which is a contradiction and establishes the lemma.

A continuity argument can now be used to establish the following.

LEMMA 2. Let $w(x)$ and $z(x)$ be solutions of $\left(5^{\prime \prime}\right)$ and $\left(6^{\prime \prime}\right)$, respectively, for which $\sigma>-\infty$ and

$$
\begin{aligned}
\tau+\int_{x_{1}}^{x}\left(\gamma-\beta^{2}\right) d t & \geqq\left|\sigma+\int_{x_{1}}^{x}\left(c-b^{2}\right) d t\right| & \text { for } x_{1} \leqq x \leqq x_{2}, \\
\beta(x) & \geqq|b(x)| & \text { for } x_{1} \leqq x \leqq x_{2} .
\end{aligned}
$$

Then $z(x) \geqq w(x)$ as long as $z(x)$ can be continued on $\left[x_{1}, x_{2}\right]$.

As an immediate consequence of Lemma 2 we have the following generalization of Levin's Theorem 1.

THEOREM 3. Suppose $u(x)$ and $v(x)$ are nontrivial solutions of $(5)$ and (6), respectively, and that $u(x) \neq 0$ for $x_{1} \leqq x<x_{2}, u\left(x_{2}\right)=0$. If

(i) $-\frac{v^{\prime}\left(x_{1}\right)}{v\left(x_{1}\right)}+\int_{x_{1}}^{x}\left(\gamma-\beta^{2}\right) d t \geqq\left|-\frac{u^{\prime}\left(x_{1}\right)}{u\left(x_{1}\right)}+\int_{x_{1}}^{x}\left(c-b^{2}\right) d t\right|$

$$
\text { for } x_{1} \leqq x \leqq x_{2} \text {, }
$$

then $v(x)$ has a zero in $\left(x_{1}, x_{2}\right]$. for $x_{1} \leqq x \leqq x_{2}$, 
Theorems 2 and 3 show how to estimate zeros of the nonselfadjoint equations (6) in terms of the coefficients and the initial value of $-v^{\prime}\left(x_{1}\right) / v\left(x_{1}\right)$. Furthermore Theorem 2 shows that such estimates are sometimes simpler in case $\tau \equiv-v^{\prime}\left(x_{1}\right) / v\left(x_{1}\right)$ is nonnegative. The following result shows how one can "shift" the initial value of $-v^{\prime}\left(x_{1}\right) / v\left(x_{1}\right)$ by means of a compensating shift in the coefficient of the differential equation.

THEOREM 4. Let $v(x)$ be a solution of (6) satisfying $-v^{\prime}\left(x_{1}\right) / v\left(x_{1}\right)=\tau$. The first zero of $v(x)$ is the same as the first zero of $V(x)$, where $V(x)$ is a solution of

$$
\begin{gathered}
V^{\prime \prime}-2\left(\beta-\tau_{0}\right) V^{\prime}+\left(c-2 \beta \tau_{0}+\tau_{0}^{2}\right) V=0, \\
-V^{\prime}\left(x_{1}\right) / V\left(x_{1}\right)=\tau+\tau_{0} .
\end{gathered}
$$

Proof. The substitution $z(x)=-v^{\prime}(x) / v(x)$ leads to

$$
z(x)=\tau+\int_{x_{1}}^{x} z^{2} d t+\int_{x_{1}}^{x} 2 \beta z d t+\int_{x_{1}}^{x} c d t .
$$

Defining $Z(x)=z(x)+\tau_{0}$ yields

$$
\begin{aligned}
Z(x) & =\tau+\tau_{0}+\int_{x_{1}}^{x}\left(Z-\tau_{0}\right)^{2} d t+\int_{x_{1}}^{x} 2 \beta\left(Z-\tau_{0}\right) d t+\int_{x_{1}}^{x} c d t \\
& =\tau+\tau_{0}+\int_{x_{1}}^{x} Z^{2} d t+\int_{x_{1}}^{x} 2\left(\beta-\tau_{0}\right) Z d t+\int_{x_{1}}^{x}\left(c-2 \beta \tau_{0}+\tau_{0}^{2}\right) d t .
\end{aligned}
$$

Now the first singularity of $Z(x)$ coincides with the first singularity of $z(x)$ and therefore with the first zero of $v(x)$. But the first singularity of $Z(x)$ also coincides with the first zero of $V(x)$ satisfying (9), by the substitution $Z(x)=-V^{\prime}(x) / V(x)$. This completes the proof.

The shift formula of Theorem 4 can also be applied in connection with other known comparison theorems for nonselfadjoint differential equations [4].

\section{BIBLIOGRAPHY}

1. A. Ju. Levin, A comparison principle for second-order differential equations, Dokl. Akad. Nauk SSSR 135 (1960), 783-786=Soviet Math. Dokl. 1 (1960), 1313-1316. MR 23 \#A1875.

2. E. Kamke, A new proof of Sturm's comparison theorems, Amer. Math. Monthly 46 (1939), 417-421. MR 1, 54.

3. C. A. Swanson, A comparison theorem for elliptic differential equations, Proc. Amer. Math. Soc. 17 (1966), 611-616. MR 34 \#1663.

4. K. Kreith, A comparison theorem for general elliptic equations with mixed boundary conditions, J. Differential Equations 8 (1970), 537-541.

Department of Mathematics, University of California, Davis, California 95616 